\title{
Long Non-Coding RNA KCNQ1OT1 Promotes Cataractogenesis via miR-214 and Activation of the Caspase-1 Pathway
}

\author{
Xin Jin ${ }^{a}$ Hao Jin ${ }^{b}$ Yan Shia Yiyuan Guo Hong Zhang ${ }^{a}$ \\ aDepartment of Ophthalmology, The First Affiliated Hospital of Harbin Medical University, Harbin, \\ 'Department of Orthopedic, The First Affiliated Hospital of Harbin Medical University, Harbin, People's \\ Republic of China
}

\section{Key Words \\ Cataract $\bullet \mathrm{KCNQ} 1 \mathrm{OT} 1 \cdot \mathrm{miR}-214 \cdot$ Caspase-1 $\cdot$ Pyroptosis}

\begin{abstract}
Background/Aims: KCNQ1OT1 regulates the expression of tissue-specific imprinted genes within the Kcnq1 domain. Imprinted genes are positive regulators of apoptosis, one of the forms of cell death related to cataract formation, and thus may provide novel therapeutic targets for cataract treatment. Here, we studied the role of non-coding RNAs(ncRNA) in cataract formation. Methods: Human lens epithelium cells (HLECs) were treated with $\mathrm{H}_{2} \mathrm{O}_{2}$. and the expression of KCNQ1OT1 and miR-214 was detected by qRT-PCR. The expression of caspase-1 was detected using qRT-PCR, western blot and immunostaining. To confirm our findings in cell cultures, we analysed KCNQ1OT1, miR-214, and caspase-1 expression in lens anterior capsules of both cataract patients and normal controls by qRT-PCR and western blot analysis. Results: We found that the expression of KCNQ1OT1 was increased in both human cataract lens anterior capsular samples and SRA01/04 cell lines treated with $\mathrm{H}_{2} \mathrm{O}_{2}$. Knockdown of KCNQ1OT1 expression significantly suppressed $\mathrm{H}_{2} \mathrm{O}_{2}$-induced SRA01/04 cell pyroptosis in vitro, which is the critical step in cataract formation. The expression of microRNA-214 (miR214) was also decreased in cataract lens anterior capsular tissues and $\mathrm{H}_{2} \mathrm{O}_{2}$-induced SRA01/04 cell lines. Knockdown of KCNQ1OT1 significantly increased the expression of miR-214. Conclusions: We demonstrated for the first time that caspase- 1 is a functional downstream target of miR-214, and knockdown of KCNQ1OT1 reduced the expression of caspase-1. These results provide evidence that the KCNQ1OT1-miR-214-caspase-1 regulatory network is a novel mechanism for promoting cataract formation.

\section{Introduction}

Age-related cataract is a cause of blindness worldwide involving genetic and environmental influences [1]. With the progress of science and technology, the surgical treatment become the most common treatment of cataract, but so far, the risk for the surgical 
complications still could not be avoided completely [2, 3].Understanding the mechanisms of cataract may help prevent cataract formation, and reduce risks of complications.

Pyroptosis is a pro-inflammatory form of cell death triggered by the inflammasome, a multi-protein complex that assembles in the cytosol to activate caspase-1. Pyroptosis is triggered by various pathological stimuli, such as brain injury [4], heart attack [5] or cancer [6], and is crucial for controlling microbial infections [7]. Our previous study has indicated that $\mathrm{H}_{2} \mathrm{O}_{2}$ can induce HLECs pyroptosis by the caspase-1 pathway. However, the complete mechanism remains unclear.

A recent study reports that non-coding RNAs are involved in the regulation of pyroptosis [8]. Non-coding RNA is an RNA molecule that is not translated into a protein. Noncoding RNAs include short non-coding RNAs and long non-coding RNAs (lncRNAs). LncRNAs, which are RNA molecules over 200 nucleotides in length, have been implicated in the regulation of a variety of cellular functions, disease processes, and cancer progression [9]. MicroRNAs (miRNAs) are a small non-coding RNA molecules found in plants, animals and some viruses that have functions in RNA silencing and post-transcriptional regulation of gene expression [10]. By binding to specific sequences, usually in the $3^{\prime}$ untranslated region (3'UTR) to degrade the transcription of target genes [11], miRNAs have been demonstrated to play an important role in cataract formation [12-15]. Although some studies have implicated IncRNAs and miRNAs in cataract formation, there is no published study to the best of our knowledge revealing the relationships between them.

In the present study, we found that IncRNA KCNQ10T1 was upregulated in cataract lens anterior capsular samples and that KCNQ10T1 knockdown inhibited the pyroptosis of HLECs. Furthermore, we demonstrated that KCNQ10T1 upregulated caspase-1 through inhibition of miR-214 expression. These results provide evidence that the KCNQ10T1-miR214-caspase-1 regulatory network promotes cataract formation.

\section{Materials and Methods}

\section{Human tissue samples}

Twenty pairs of lens anterior capsular samples were obtained from cataract patients (twenty patients, free of other ocular diseases) at the operating room of the Eye Hospital of Harbin Medical University, China. Control lens anterior capsular samples were collected from healthy postmortem eyes (twenty donors, free of ocular diseases) donated to the eye bank of Heilongjiang Province. Samples were immediately snap-frozen and stored at $-80^{\circ} \mathrm{C}$ until RNA extraction. All samples were collected with informed consent obtained from all the patients, and the study was approved by the Research Ethics Committee of Harbin Medical University.

\section{Cell culture and transduction}

SV40 T-antigen-transformed human LEC line (SRA01/04 cell) were cultured in Dulbecco's modified DMEM/F12 supplemented with $20 \%$ foetal bovine serum (FBS) at $37^{\circ} \mathrm{C}$ in a $5 \% \mathrm{CO}_{2}$ atmosphere incubator overnight, and the cells were treated with the different concentrations of $\mathrm{H}_{2} \mathrm{O}_{2}$ for the required time. SiRNAs of human KCNQ10T1 were constructed by RIBOBIO (Guangzhou, China). The overexpression and knockdown of miR-214 were provided by Invitrogen (Carlsbad, CA, USA). All cell transfections were performed according to the manufacturer's protocol.

\section{RNA extraction and quantitative PCR}

Total RNA was extracted using TRIzol reagent (Invitrogen, Carlsbad, CA, USA). PCR primers were purchased from Invitrogen (Carlsbad, CA). The concentration of RNA was determined using a NanoDrop Spectrophotometer (NanoDrop Technologies, Wilmington, DE). For detection of miR-214 expression level, cDNA was synthesized from $5 \mathrm{ng}$ of total RNA using the TaqMan ${ }^{\circledR}$ miRNA reverse transcription kit (Applied Biosystems, Foster City, CA, USA). The relative miR-214 expression levels after normalization to U6 small nuclear RNA was calculated using the $2^{-\Delta \Delta \mathrm{Ct}}$ method.

For detection of caspase-1 mRNA expression, cDNAs were synthesized from total RNA using gene specific primers from a cDNA synthesis kit (HIGH-Capacity cDNA Reverse Transcription Kits, USA). 


\section{Cellular Physiology Cell Physiol Biochem 2017;42:295-305 \begin{tabular}{l|l|l} 
and Biochemistry $\begin{array}{l}\text { DOI: 10.1159/000477330 } \\
\text { Published onIne: May 22, } 2017\end{array}$ & $\begin{array}{l}\text { @ } 2017 \text { The Author(s). Published by S. Karger AG, Basel } \\
\text { www.karger.com/cpb }\end{array}$
\end{tabular} \\ Jin et al.: Long Non-Coding RNA KCNQ1OT1 Promotes Cataractogenesis}

Quantitative real-time PCR was performed using SYBR Green Real-Time PCR MasterMix (ToYoBo, Japan) according to the manufacturer's protocols using an ABI 7500 Sequence Detection System (Life Technologies, NY, USA). PCR amplification was performed in a 20- $\mu$ l reaction volume containing $2 \mu \mathrm{l} \mathrm{cDNA,6} \mu \mathrm{l}$ DEPC,10 $\mu \mathrm{l}$ STBR Master Mix, $1 \mu \mathrm{l}$ forward primer and $1 \mu \mathrm{l}$ reverse primer. PCR conditions were as follows: holding $95^{\circ} \mathrm{C}$ for $60 \mathrm{~s}, 40$ cycles at $95^{\circ} \mathrm{C}$ for $15 \mathrm{~s}, 60^{\circ} \mathrm{C}$ for $15 \mathrm{~s}, 72^{\circ} \mathrm{C}$ for $45 \mathrm{~s}$. Glyceraldehyde 3-phophate dehydrogenase (GAPDH) was used as an internal control. The relative caspase-1 expression level was normalized to GAPDH using the $2^{-\Delta \Delta \mathrm{Ct}}$ method. The primer sequences are listed in Table 1.

\section{Western blot analysis}

Western blot analysis was used to detect the expression levels of proteins of interest. Protein (50 $\mu \mathrm{g}$ ) per sample was separated using 10\% SDS-PAGE and then transferred onto nitrocellulose membranes (USA). The membrane was blocked with 5\% non-fat milk (BD Biosciences) and 0.1\% Tween 20 in trisbuffered saline, then immunoblotted overnight using appropriate primary antibodies at $4{ }^{\circ} \mathrm{C}$ with gentle shaking. Fluorochrome-labelled secondary antibody (Alexa Fluor 800, LI-COR, USA) was used to identify the appropriate primary antibody. Immunoreactivity was detected with the Odyssey fluorescent scanning system (LI-COR) and analysed by Image Studio software. GAPDH was used as a loading control.

\section{Cell proliferation assay}

Cell proliferation assay kits (CCK-8) were used in accordance with the manufacturer's instructions. HLECs were seeded in 96-well plates at $1 \times 10^{4}$ cells/well and maintained for $24 \mathrm{~h}$. CCK-8 solution (10 $\mu \mathrm{l}$ ) was added to each well, and cells were incubated in $37^{\circ} \mathrm{C}$ for $2 \mathrm{~h}$. The absorbance (A) at $450 \mathrm{~nm}$ was evaluated using a microplate reader. The data are representative of three individual experiments in performed in triplicate.

\section{Immunofluorescence staining}

SRA01/04 cells treated with siNC and siKCNQ10T1 were fixed with 4\% paraformaldehyde for 20 minutes at room temperature. After rinsing with PBS, cells were blocked with 5\% BSA. The cells were incubated with rabbit caspase-1 (1:200; Sigma) overnight at $37^{\circ} \mathrm{C}$. After rinsing three times in PBS, cells were incubated with the corresponding secondary antibody, Cy3-conjugated anti-rabbit IgG (1:100; Sigma). The nuclei were stained with DAPI.

\section{TUNEL staining}

SRA01/04 cells were plated onto coverslips in 24-well culture plates, and In Situ Cell Death Detection Kits (TUNEL fluorescence FITC kit, Roche, Indianapolis, IN, USA) were employed to detect DNA fragmentation of individual cells according to the manufacturer's instructions. The nuclei were stained with DAPI. TUNEL staining was assessed via fluorescence microscopy (Eclipse 80i; Nikon Co., Tokyo, Japan). Nuclei double labeled with DAPI and TUNEL were considered positive.

\section{Luciferase assay}

The miR-214 binding sites of KCNQ10T1 and caspase-13'-UTR were predicted using the bioinformatics databases (Starbase v2.0, miRcode and RNAhybrid). The CASP1 3'-UTR and KCNQ10T1 3'-UTR luciferase reporter vector was purchased from RIBOBIO (Guangzhou, China). Restriction sites for XhoI and NotI were added to forward and reverse primers (IDT DNA), respectively. To examine the relationship between miR-214 and CASP1, we constructed wild-type and mutated-type CASP1 3'-UTR reporter plasmid with psiCheck-2 vector. To test whether miR214 targets KCNQ10T1 directly, the PCR amplified KCNQ10T1 3'-UTR(wild-type and mutatedtype) were cloned into the pmiR-

Table 1. The primers used for qRT-PCR

\begin{tabular}{|c|c|c|}
\hline Names & Sequence $\left(5^{\prime}-3^{\prime}\right)$ & Length (bp) \\
\hline \multirow[t]{2}{*}{ G A P D H } & A A G A A G G T G T G A A G C A G G C & 20 \\
\hline & T C C A C C ACC C T G T T G C T G TA & 20 \\
\hline \multirow[t]{2}{*}{ C A S P 1} & A CACG T C T T G C C C T CATTATCT & 22 \\
\hline & A T A A C C T T G G G C T T G T C T T T C A & 22 \\
\hline \multirow[t]{2}{*}{ U 6} & C T C G C T T C G G C A G C A C A T A T A C T & 23 \\
\hline & A C G C T T C A C G A A T T T G C G T G T C & 22 \\
\hline \multirow[t]{2}{*}{ m i R - 214} & TATACATCAAACAGCAGGCACA & 22 \\
\hline & C ATTCGATCT TCTCCACAGTCTC & 23 \\
\hline
\end{tabular}




\section{Cellular Physiology Cell Physiol Biochem 2017;42:295-305

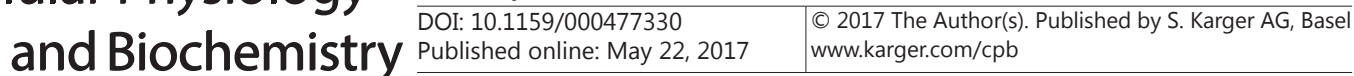

Jin et al.: Long Non-Coding RNA KCNQ1OT1 Promotes Cataractogenesis

RB-Report vector. The luciferase activity was measured 48 hours after transfection using the Dual-Luciferase Reporter Assay System(Promega).

Statistical analysis

Statistical significance was determined using Student's t-test (two-tailed), and one-way ANOVA. Results were expressed as the mean \pm standard deviation (SD). The differences were considered to be statistically significant at $\mathrm{P}<0.05$.

\section{Results}

KCNQ10T1 and caspase-1 involved in cataract formation

We first investigated the IncRNA KCNQ10T1 expression level in both cataract and normal capsule tissues by qRT-PCR. The KCNQ10T1 levels were higher in cataract lens anterior capsules than normal controls (Fig. 1A). We also evaluated lncRNA KCNQ10T1 expression level in SRA01/04 cells treated with different concentrations of $\mathrm{H}_{2} \mathrm{O}_{2}(0,25,50$ and100 $\mu \mathrm{M})$. Our results showed that KCNQ10T1 levels were increased in a dose-dependent manner with $\mathrm{H}_{2} \mathrm{O}_{2}$ concentration (Fig. 1B). We also measured the mRNA and protein expression of caspase- 1 both in tissues and in SRA01/04 cells treated with the same concentrations of $\mathrm{H}_{2} \mathrm{O}_{2}$ as above. The mRNA and protein expression levels of caspase- 1 were also significantly upregulated in cataract samples compared to normal controls (Fig. 1C-D). At the same time, caspase- 1 levels were higher with the increase of $\mathrm{H}_{2} \mathrm{O}_{2}$ concentration in SRA01/04 cells (Fig. $1 \mathrm{E}-\mathrm{F})$. These findings imply that KCNQ10T1 and caspase-1 levels have a strong correlation with cataract formation.

Validation of pyroptosis in SRA01/04 cells treated with $\mathrm{H}_{2} \mathrm{O}_{2}$

Caspase- 1 is a commonly utilized marker of pyroptosis. To detect whether pyroptosis is involved in cataract formation, SRA01/04 cells were exposed to various concentrations of $\mathrm{H}_{2} \mathrm{O}_{2}(0,25,50$ and $100 \mu \mathrm{M})$ for $24 \mathrm{~h}$. Pyroptosis was detected by CCK-8, immunofluorescence staining and TUNEL staining (Fig. 2A-C). SRA01/04 cell growth was inhibited by 100 and $200 \mu \mathrm{M} \mathrm{H}_{2} \mathrm{O}_{2}$ at 24 and $48 \mathrm{~h}$, as shown by the CCK-8 assay (Fig. 2A). Immunofluorescence staining showed much stronger positive staining of caspase- 1 in SRA01/04 cells treated with $100 \mu \mathrm{M} \mathrm{H}_{2} \mathrm{O}_{2}$ (Fig. 2B). The TUNEL assay also showed that $\mathrm{H}_{2} \mathrm{O}_{2}$-induced cell pyroptosis was significantly increased in a dose-dependent manner (Fig. 2C). Based on these results, we chose $100 \mu \mathrm{M} \mathrm{H}_{2} \mathrm{O}_{2}$ for pyroptosis induction in our subsequent experiments.

KCNQ1OT1 knockdown inhibits the pyroptosis of SRA01/04 cells

We next examined the role of KCNQ10T1 in the pyroptosis of HLECs treated with 100 $\mu \mathrm{M} \mathrm{H}_{2} \mathrm{O}_{2}$. After demonstrating the effectiveness of KCNQ10T1 siRNAs (Fig. 3A), pyroptosis in HLECs was determined using CCK-8 and Tunel staining. The CCK-8 assay results showed that KCNQ10T1 knockdown reduced HLECs pyroptosis compared with cells transfected with control siRNA (Fig. 3B). After successful transfection, pyroptosis was significantly reduced, as shown by Tunel staining (Fig. 3C). As shown in Fig. 3B-C, both CCK-8 and Tunel staining showed that KCNQ10T1 siRNAs could reduce pyroptosis in HLECs. These data suggest that KCNQ10T1 contributes to HLECs pyroptosis in vitro. Moreover, we measured caspase-1 expression levels between HLECs transfected with KCNQ10T1 siRNAs and siNC. Our results show that HLECs transfected with KCNQ10T1 siRNAs had lower caspase-1 expression (Fig. 3D-F).

miR-214 as a potential factor in KCNQ10T1-caspase-1 pathway

In the above experiments, we found that KCNQ10T1 expression was correlated with caspase-1. To elucidate this mechanism, we investigated potential miRNAs. We used bioinformatics tools (MicroRNA, Starbase v2.0) to examine the potential complementary base pairing between KCNQ10T1 and miRNAs. We found that miR-214 contains potential 
A

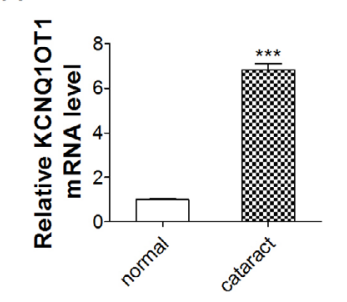

D
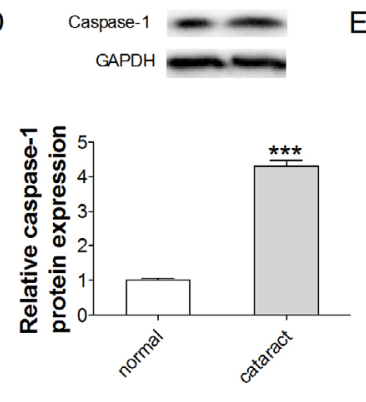

B

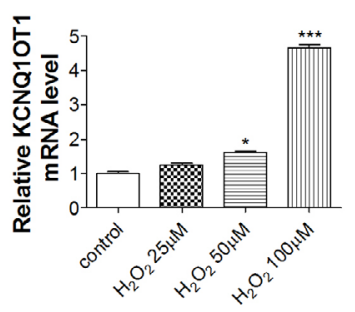

E

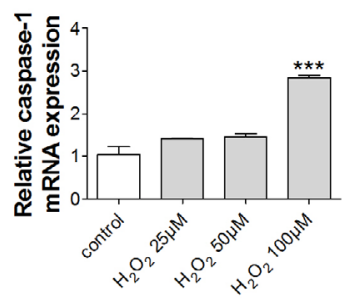

C

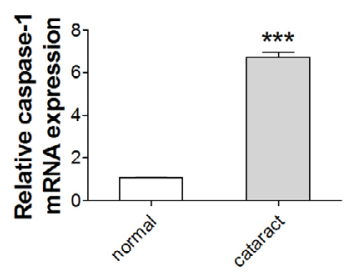

F
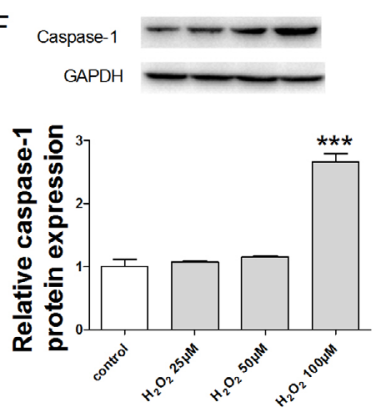

Fig. 1. KCNQ10T1 and caspase-1 involved in cataract formation. A: qRT-PCR results of the relative mRNA levels of KCNQ10T1 in anterior lens capsules of normal donors and cataract patients; B: qRT-PCR results showed that the relative KCNQ10T1 mRNA levels was increased with the increased concentration of $\mathrm{H}_{2} \mathrm{O}_{2}(0,25,50,100 \mu \mathrm{M})$; ( $\mathrm{C}$ and $\left.\mathrm{D}\right)$ : The mRNA and protein levels of caspase- 1 in anterior lens capsules of normal donors and cataract patients; (E and F): qRT-PCR and western-blot results showed that the relative caspase- 1 levels was increased with the increased concentration of $\mathrm{H}_{2} \mathrm{O}_{2}(0,25,50,100 \mu \mathrm{M})$; Data are expressed as mean \pm SEM. $\mathrm{n}=3$. ${ }^{*} \mathrm{P}<0.05,{ }^{* * *} \mathrm{P}<0.001$ versus control.

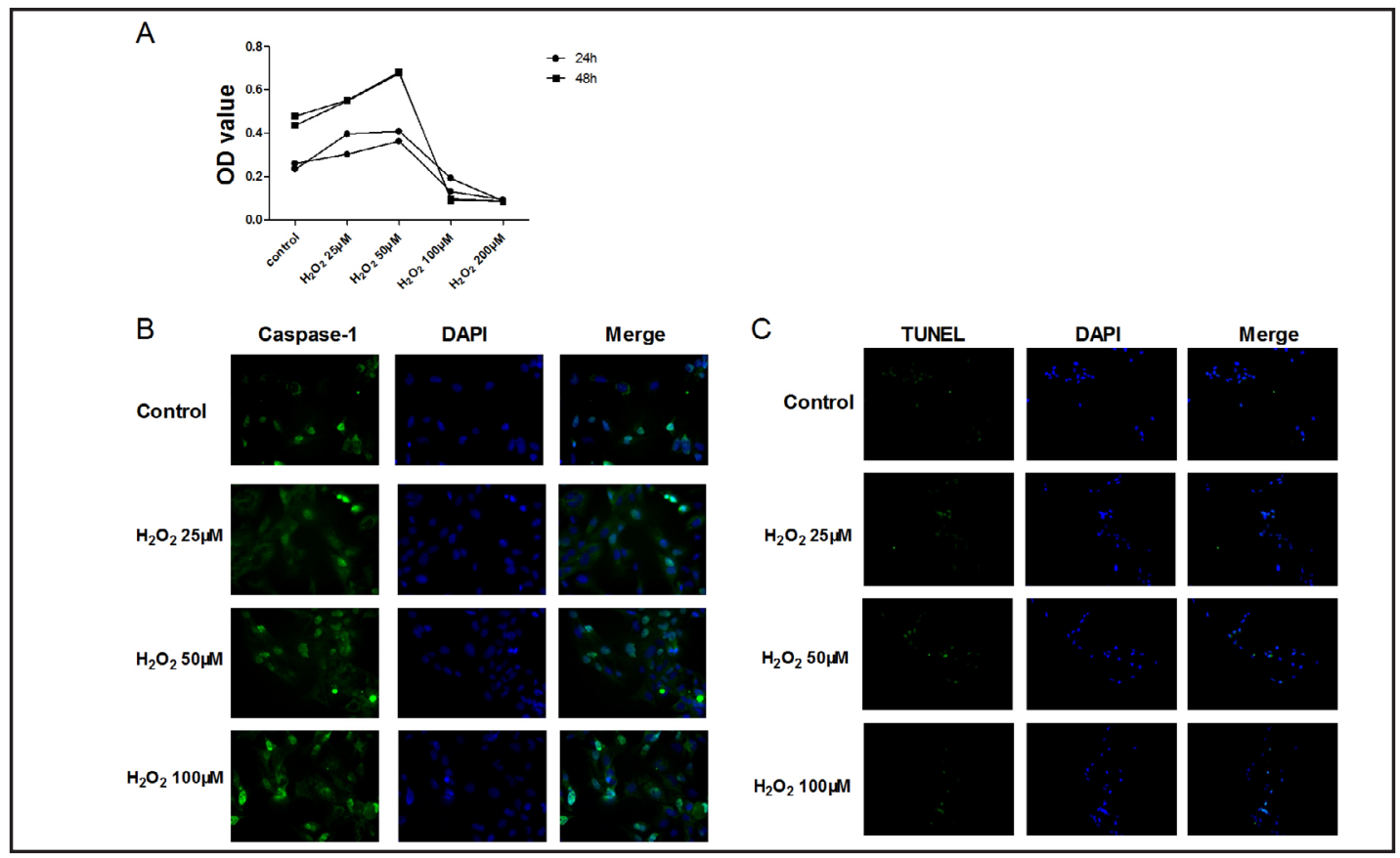

Fig. 2. Validation of pyroptosis in SRA01/04 cells treated with $\mathrm{H}_{2} \mathrm{O}_{2} \mathrm{~A}$ : CCK-8 assay results revealed that SRA01/04 cells growth was inhibited when co-cultured with 100,200 $\mu_{\mathrm{M} \mathrm{H}} \mathrm{O}_{2}$ for 24, $48 \mathrm{~h}$; B: Immunofluorescence images showing the expression of caspase- 1 in SRA01/04 cells treated with $\mathrm{H}_{2} \mathrm{O}_{2}(0,25,50,100 \mu \mathrm{M})$; Blue: nuclear staining (DAPI); Green: caspase-1staining. Scalebar:20 $\mu \mathrm{m}$. C: TUNEL images of SRA01/04 cells. Cells were treated with $0 \mu \mathrm{M}, 25 \mu \mathrm{M}, 50 \mu \mathrm{M}$ and $100 \mu \mathrm{M} \mathrm{H}_{2} \mathrm{O}_{2}$, respectively. Blue, nuclear staining (DAPI); Green,TUNEL staining .Scalebar: $20 \mu \mathrm{m}$. 


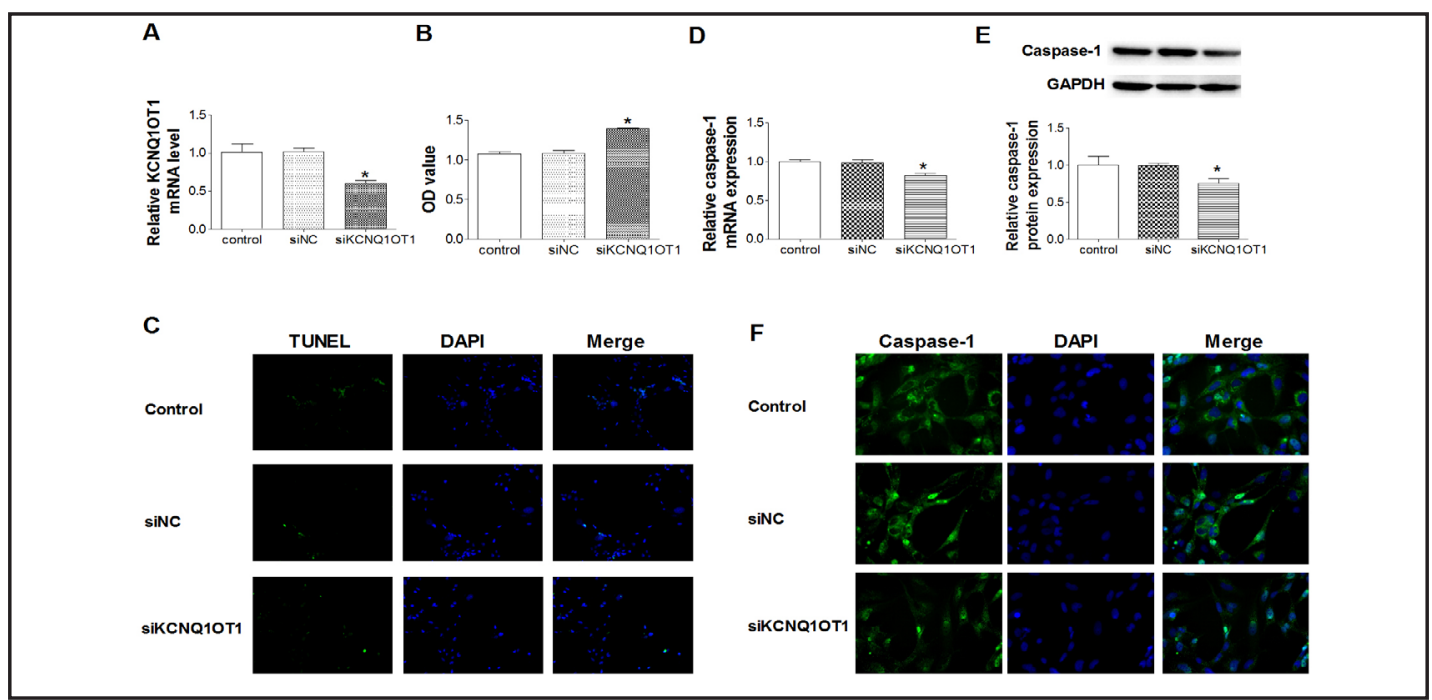

Fig. 3. KCNQ10T1 knockdown inhibits the pyroptosis of SRA01/04 cells A: Relative mRNA levels of KCNQ10T1 of SRA01/04 cells in the control, siNC, siKCNQ10T1 groups. B: Effect of siKCNQ10T1 on proliferation of SRA01/04 cells by CCK-8 assay. C: TUNEL images of SRA01/04 cells of the control, siNC, siKCNQ10T1 groups. Blue, nuclear staining (DAPI); Green, TUNEL staining. Scalebar: $20 \mu \mathrm{m}$. (D and E): Relative mRNA and protein levels of caspase- 1 of SRA01/04 cells in the control, siNC, siKCNQ10T1 groups. F: Immunofluorescence images showing the expression of caspase-1 in SRA01/04 cells of the control, siNC, siKCNQ10T1 groups. Blue: nuclear staining (DAPI); Green: caspase-1 staining. Scalebar: $20 \mu \mathrm{m}$.Data are expressed as mean \pm SEM. $n=3 . * P<0.05,{ }^{* * *} \mathrm{P}<0.001$ versus control.

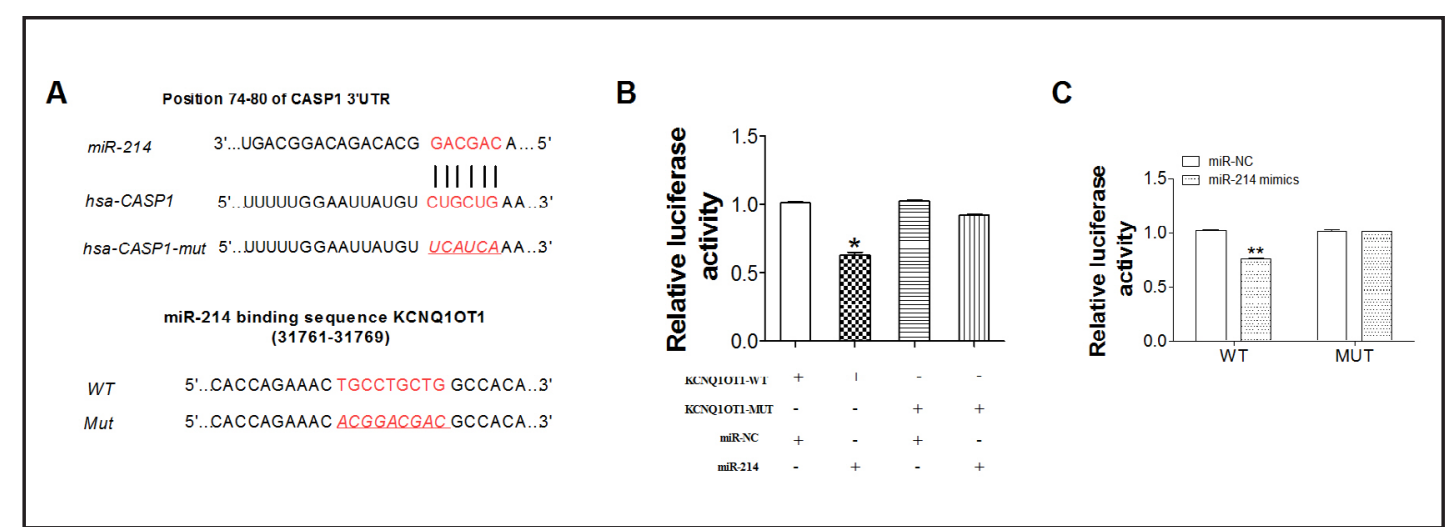

Fig. 4. miR-214 as a potential factor in KCNQ10T1-caspase-1 pathway. A: Bioinformatics predicted miR214 binding sites in CASP1. Partial sequences of miR-214 and binding sites in the CASP1 3'-UTR are shown. "CUGCUG" in WT CASP1 3'-UTR turn into "UCAUCA" in MUT CASP1 3'-UTR as red line. Bioinformatics predicted miR-214 binding sites in KCNQ10T1 3'-UTR. The sequences of KCNQ10T1 WT and mutant are shown below in Fig.4A. B: Co-transfection with WT KCNQ10T1 3'-UTR, miR-214 mimics ,miR-NC or MUT KCNQ10T1 3'-UTR,miR-214 mimics,miR-NC are showed middle in Fig.4B. C:Luciferase reporter plasmids containing WT or mutant CASP1 3'-UTR were co-transfected into SRA01/04 cells with miR-214 mimics in parallel with miR-NC. The data are presented as the relative luciferase activity.

binding sites for both KCNQ10T1 and caspase-1 3'-UTR (Fig. 4A). Then, we used a luciferase assay system to test whether these bioinformatics analysis results are correct. As shown in Fig. 4B-C, the relative luciferase activities in cells transfected with WT KCNQ10T1-3'-UTR were significantly reduced in the presence of miR-214. Co-transfection with WT caspase-1 3'-UTR and miR-214 mimics the significantly reduced luciferase reporter activity compared with cells transfected with MUT caspase-1 3'-UTR and miR-214 mimics. 
A

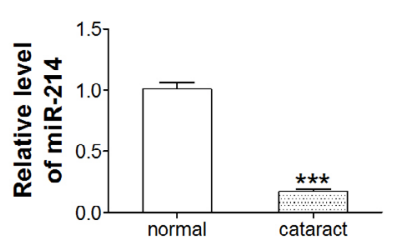

D

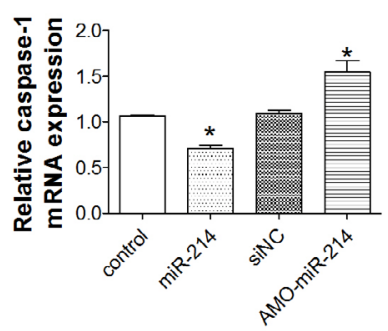

B

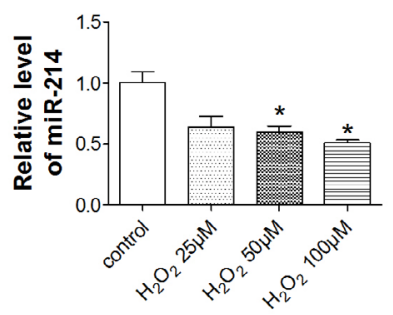

C

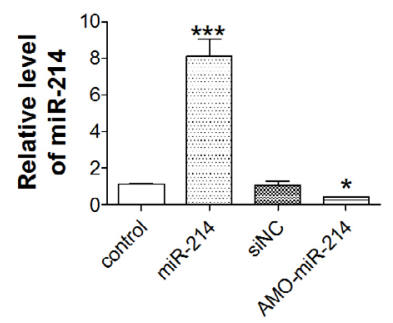

E

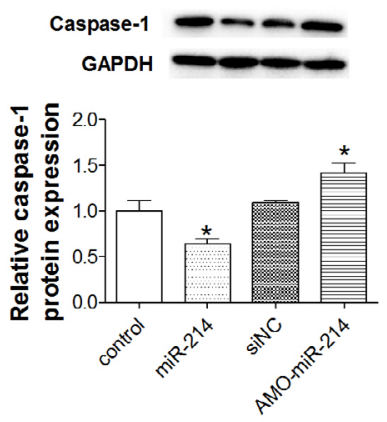

Fig. 5. miR-214 in cataract A: The miR-214 levels in anterior lens capsules of normal donors and cataract patients; B: qRT-PCR results showed that the relative miR-214 levels was reduced with the increased concentration of $\mathrm{H}_{2} \mathrm{O}_{2}$; C: The miR-214 levels in SRA01/04 cells transfected with control, miR-214, siNC and AMO-miR-214 by qRT-PCR. (D and E): Relative mRNA and protein levels of caspase-1 of SRA01/04 cells in the control, miR-214, siNC,AMO-miR-214 groups. Data are expressed as mean \pm SEM. $n=3$. ${ }^{*} \mathrm{P}<0.05$, $* * * \mathrm{P}<0.001$ versus control.

miR-214 in cataract

Our next experiments were performed to confirm whether miR-214 is involved in cataract formation. We detected miR-214 expression in both cataract lens anterior capsules and in HLECs treated with $\mathrm{H}_{2} \mathrm{O}_{2}$. As shown in (Fig. 5A), the relative miR-214 levels were lower in the cataract lens anterior capsule than normal lens capsule tissues. Similarly, relative miR-214 levels decreased in HLECs with the increases in $\mathrm{H}_{2} \mathrm{O}_{2}$ concentration (Fig. 5B). To further investigate whether miR-214 was a functional target of KCNQ10T1, we first examined miR-214 expression levels in HLECs transfected with KCNQ10T1-siRNA or a negative control (siNC). qRT-PCR results showed that after treatment with $100 \mu \mathrm{M} \mathrm{H}_{2} \mathrm{O}_{2}$, miR-214 expression was elevated in HLECs transfected with KCNQ10T1-siRNA compared with HLECs transfected with siNC (Fig. 6A). To confirm caspase-1 is the downstream target of miR-214, we first demonstrated the effectiveness of miR-214 transfection (Fig. 5C). After pretreatment with $100 \mu \mathrm{M} \mathrm{H}_{2} \mathrm{O}_{2}$, HLECs were transfected with control, miR-214mimic, siNC and AMO-miR-214. We found that overexpression of miR-214 abrogated the increase of caspase-1, and the opposite result occurred in AMO-miR-214 (Fig. 5D-E). Furthermore, inhibition of KCNQ10T1 overcame the increase of caspase-1 by miR-214 inhibitor (Fig. $6 \mathrm{~A}-\mathrm{B})$. All these results suggest an important role of KCNQ10T1 in regulating caspase- 1 by competitively binding miR-214.

miR-214 reverses the accelerative effects of KCNQ1OT1 in HLECS

Our results demonstrate that miR-214 is a target of KCNQ10T1(Fig. 4A,6A), but the role of miR-214 in KCNQ10T1-mediated effects in HLECs remained unclear. Our next experiments were designed to determine whether KCNQ10T1 might promote pyroptosis in HLECs via the potential KCNQ10T1-miR-214-caspase-1 pathway. qRT-PCR assays revealed that the 


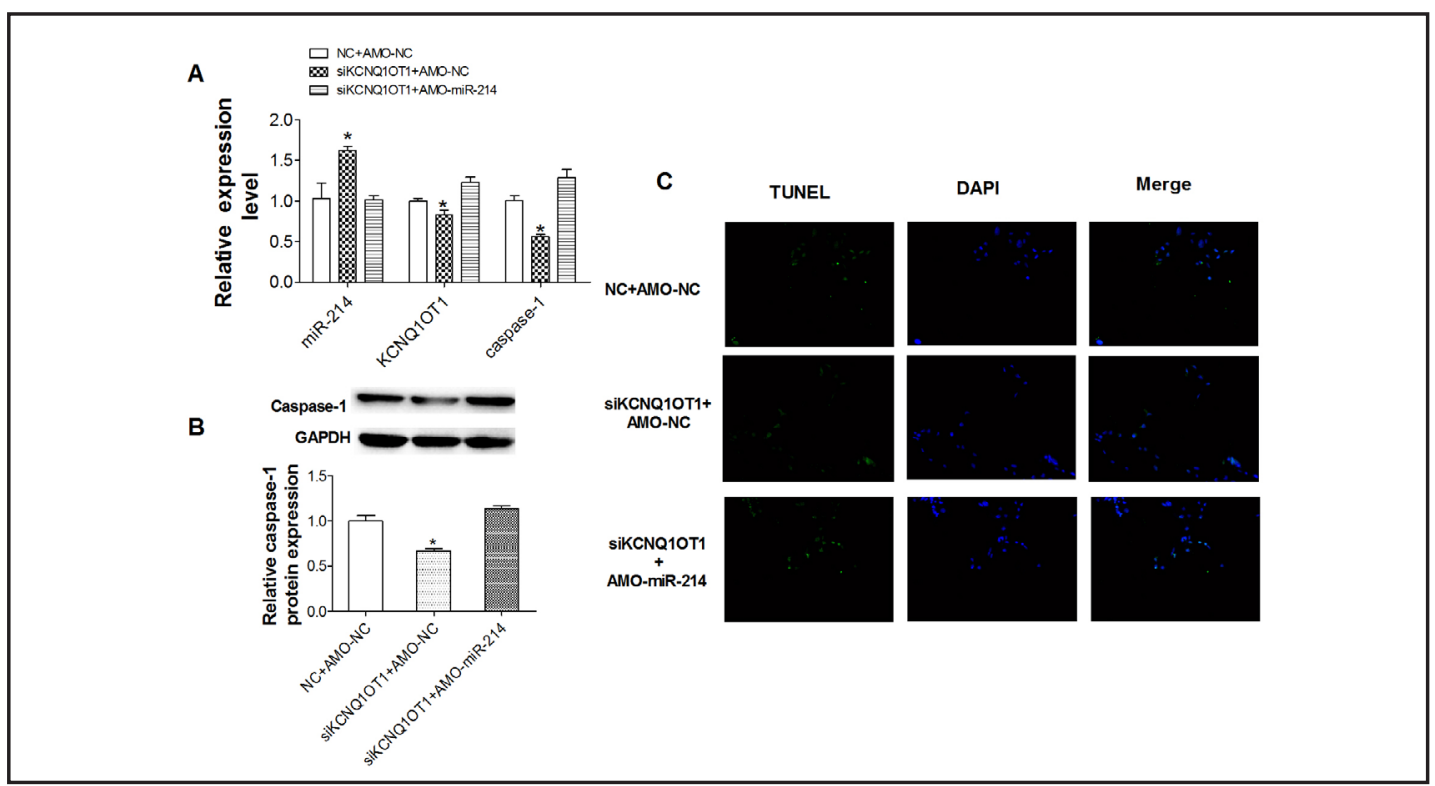

Fig. 6. miR-214 reverses the acceerative effects of KCNQ10T1 in SRA01/04 cells. A: miR-214 expression was visibly elevated in SRA01/04 cells transfected with siKCNQ10T1. The caspase-1 mRNA expression levels are reduced by inhibition of KCNQ10T1 and reversed by miR-214 inhibitor. B: The caspase-1 protein expression levels are in accordance with its mRNA expression levels. C: TUNEL images of SRA01/04 cells treated with $100 \mu \mathrm{M} \mathrm{H}_{2} \mathrm{O}_{2}$ then transfected with NC+AMO-NC, siKCNQ10T1+AMO-NC, siKCNQ10T1+AMO-miR-214 groups. Blue, nuclear staining (DAPI); Green, TUNEL staining. Scalebar: $20 \mu \mathrm{m}$. Data are expressed as mean \pm SEM. $n=3 .{ }^{*} \mathrm{p}<0.05$ when compared with $\mathrm{NC}+\mathrm{AMO}-\mathrm{NC}$ group.

decrease of KCNQ10T1 expression by KCNQ10T1-siRNA could be largely reversed by miR214 (Fig. 6A). We used Tunel staining to correlate cell death with caspase- 1 expression. $\mathrm{H}_{2} \mathrm{O}_{2}$ induced cell pyroptosis was reduced by inhibition of KCNQ10T1 and reversed by miR-214 inhibition (Fig. 6C). Taken together, our results suggest that KCNQ10T1 promotes pyroptosis in HLECs through the KCNQ10T1-miR-214-caspase-1 axis and that miR-214 could reverse the influence of KCNQ10T1 on HLECs.

\section{Discussion}

Recent studies have demonstrated the functional roles of IncRNAs and provided insights into the molecular mechanisms by which lncRNAs function in a variety of human diseases [16]. Several studies have proposed that mRNAs and lncRNAs can regulate each other through their capacity to compete for miRNA binding $[17,18]$. In this study, for the first time, we reported that KCNQ10T1 plays an important role in cataract formation. KCNQ10T1 was highly regulated in $\mathrm{H}_{2} \mathrm{O}_{2}$-treated HLECs and cataract lens anterior capsule tissues, and its overexpression promotes pyroptosis in HLECs, possibly by targeting and inhibiting miR-214.

KCNQ10T1 encodes a paternally expressed IncRNA that has been shown to regulate imprinting of several genes present at 11p15.5 locus in cis [19]. KCNQ10T1 might inhibit tumour development and/or progression in many diseases, including colorectal cancer [20] and lung cancer [21]. At the same time, KCNQ10T1 regulates the expression of both ubiquitous and tissue-specific imprinted genes within the Kcnq1 domain [22]. Imprinted genes are a positive regulator of apoptosis and tumour suppression [23, 24]. Apoptosis of lens epithelial cells is a common cellular mechanism of initiation and progression for noncongenital cataracts [25]. Apoptosis is the most widely recognized programme of cell death [26], but other cell death programmes, such as autophagy, oncosis and pyroptosis, are also 
involved in occurrence and development of diseases [27, 28]. Our previous studies indicate that pyroptosis is implicated in the regulation of cataract formation. However, the role of KCNQ10T1 in regulating cataracts has remained unknown.

Mir-214 is involved in various physiological processes, such as melanoma tumours [29], uveal melanoma [30], differentiation of stem cells [31], gastric cancer [32], and arterial hypertension [33]. However, the biological functions of miR-214 in cataracts have not been completely elucidated. Our study provides new evidence that highly overexpressed KCNQ10T1 in cell lines and cataract lens anterior capsule tissues acts in a monogenic role. We also found that KCNQ10T1 is a specific target of miR-214, and there is an interactive suppression between KCNQ10T1 and miR-214. Our data suggest that KCNQ10T1 may promote pyroptosis in cataracts due to its ability to inhibit miR-214 and subsequent activation of caspase- 1 . Caspase- 1 is an important marker in the development of pyroptosis [34]. In this study, we found that KCNQ10T1 expression in normal lens anterior capsule tissues is remarkably lower than that in cataract lens anterior capsule tissues. Our observations demonstrate KCNQ10T1 could play a critical role in cataract development and progression. However, the underlying mechanism by which KCNQ10T1-mediated gene expression remains to be elucidated. Several studies report that IncRNAs can function as competing endogenous RNAs to regulate microRNAs in various cellular processes $[35,36]$. We discovered that KCNQ10T1 may function as endogenous miRNA "sponge" to bind miR214 and regulate its function. We detected miR-214 expression in HLECs with increased $\mathrm{H}_{2} \mathrm{O}_{2}$ concentration, and in cataract lens anterior capsule tissues compared to normal controls. KCNQ10T1 and miR-214 showed an opposite association, suggesting regulatory relationship between miR-214 and KCNQ10T1 in pyroptosis and cataract formation. We found that miR214 overexpression decreased caspase-1 levels in HLECs. Furthermore, our data suggests there are miR-214 binding sites on KCNQ10T1 sequences that miR-214 directly binds.

Taken together, this study supports the hypothesis that KCNQ10T1 may induce pyroptosis through the miR-214/caspase- 1 pathway during cataract formation. This report reveals a novel mechanism of KCNQ10T1 in cataract formation. KCNQ10T1 can directly interact with miR-214, lowering its expression and thus modulating caspase-1 downstream. These studies may provide a basis for developing novel therapeutic strategies for cataract prevention and treatment in the future.

\section{Acknowledgements}

Supported by the National Natural Science Foundation of China 81671844; Distinguished Young Scientists Foundation of Heilongjiang Province JC2016019; Provincial Subject Research Institutes Foundation 201608; Translational Medicine Medical Research Center Special Fund Project CR201407.

\section{Disclosure Statement}

The authors declare that there is no conflict of interests regarding the publication of this paper.

\section{References}

1 Harocopos GJ, Alvares KM, Kolker AE, Beebe DC: Human age-related cataract and lens epithelial cell death. Invest Ophthalmol Vis Sci 1998;39:2696-706.

2 Meacock WR, Spalton DJ, Boyce J, Marshall J: The effect of posterior capsule opacification on visual function. Invest Ophthalmol Vis Sci 2003;44:4665-4669.

-3 Fernandez V, Fragoso MA, Billotte C, Lamar P, Orozco MA, Dubovy S: Efficacy of various drugs in the prevention of posterior capsule opacification: experimental study of rabbit eyes. J Cataract Refract Surg 


\section{Cellular Physiology Cell Physiol Biochem 2017;42:295-305

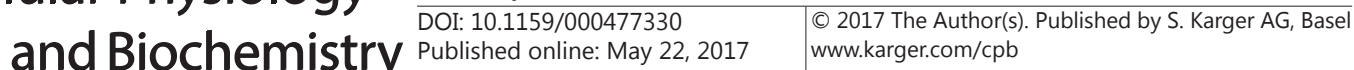

Jin et al.: Long Non-Coding RNA KCNQ1OT1 Promotes Cataractogenesis

2004;30:2598-2605.

4 Yang F, Wang Z, Wei X: NLRP3 deficiency ameliorates neurovascular damage in experimental ischemic stroke. J Cereb Blood Flow Metab 2014;3:660-667.

5 Li X, Du N, Zhang Q: MicroRNA-30d regulates cardiomyocyte pyroptosis by directly targeting foxo3a in diabetic cardiomyopathy. Cell Death Dis 2014;5:e1479.

6 Hu B, Elinav E, Huber S, Booth CJ, Strowig T, Jin C: Inflammation-induced tumorigenesis in the colon is regulated by caspase-1 and NLRC4. Proc Natl Acad Sci USA 2010;107:21635-21640.

-7 Fann DY, Lee SY, Manzanero S, Tang SC, Gelderblom M, Chunduri P: Intravenous immunoglobulin suppresses NLRP1 and NLRP3 inflammasome-mediated neuronal death in ischemic stroke. Cell Death Dis 2013;4:e790.

-8 Lee S, Choi E, Cha MJ: Looking for Pyroptosis-Modulating miRNAs as a Therapeutic Target for Improving Myocardium Survival. Mediators Inflamm 2015;2015:254871.

-9 Chen LL and Zhao JC: Functional analysis of long noncoding RNAs in development and disease. Adv Exp Med Biol 2014;825:129-158.

10 Zhou S, Li M, Zeng D, Xu X, Fei L, Zhu Q, Zhang Y, Wang R: A single nucleotide polymorphism in 3' untranslated region of epithelial growth factor receptor confers risk for pulmonary hypertension in chronic obstructive pulmonary disease. Cell Physiol Biochem 2015;36:166-178.

11 Carrillo ED, Sampieri R, Hernández A, García MC, Sánchez JA: MiR-132 Regulates Rem Expression in Cardiomyocytes During Long-Term $\beta$-Adrenoceptor Agonism. Cell Physiol Biochem 2015;36:141-54.

$\checkmark 12$ Chien KH, Chen SJ, Liu JH: Correlation between microRNA-34a levels and lens opacity severity in agerelated cataracts.Eye (Lond) 2013;27:883-888.

13 Dong N, Tang X: miRNA-181a inhibits the proliferation, migration, and epithelial-mesenchymal transition of lens epithelial cells. Invest Ophthalmol Vis Sci 2015;56:993-1001.

-14 Dong N, Xu B, Benya SR: MiRNA-26b inhibits the proliferation, migration, and epithelial-mesenchymal transition of lens epithelial cells. Mol Cell Biochem 2014;396:229-238.

15 Li Y, Liu S, Zhang F: Expression of the microRNAs hsa-miR-15a and hsa-miR-16-1 in lens epithelial cells of patients with age-related cataract. Int J Clin Exp Med 2015;8:2405-2410.

16 Wang D, Wang D, Wang N: Long Non-Coding RNA BANCR Promotes Endometrial Cancer Cell Proliferation and Invasion by Regulating MMP2 and MMP1 via ERK/MAPK Signaling Pathway. Cell Physiol Biochem 2016;40:644-656.

17 Zhao L, Han T, Li Y: The IncRNA SNHG5/miR-32 axis regulates gastric cancer cell proliferation and migration by targeting KLF4. FASEB J 2017;31:893-903.

18 He GY, Hu JL, Zhou L:The FOXD3/miR-214/MED19 axis suppresses tumour growth and metastasis in human colorectal cancer. Br J Cancer 2016;115:1367-1378.

19 Mohammad F, Pandey GK, Mondal T, Enroth S, Redrup L, Gyllensten U, Kanduri C: Long noncoding RNA-mediated maintenance of DNA methylation and transcriptional gene silencing. Development2012;139:2792-2803.

20 Nakano S, Murakami K, Meguro M, Soejima H, Higashimoto K, Urano T, Kugoh H, Mukai T, Ikeguchi M, Oshimura M: Expression profile of LIT1/KCNQ10T1 and epigenetic status at the KvDMR1 in colorectal cancers. Cancer Sci 2006;97:1147-1154.

-21 Soejima H, Nakagawachi T, Zhao W, Higashimoto K, Urano T, Matsukura S, Kitajima Y, Takeuchi M, Nakayama M, Oshimura M: Silencing of imprinted CDKN1C gene expression is associated with loss of CpG and histone H3 lysine 9 methylation at DMR-LIT1 in esophageal cancer. Oncogene 2004;23:4380-4388.

22 Mohammad F, Mondal T, Guseva N: Kcnq1ot1 noncoding RNA mediates transcriptional gene silencing by interacting with Dnmt1. Development 2010;137:2493-2499.

23 Murakami K, Oshimura M: Suggestive evidence for chromosomal localization of non-coding RNA from imprinted LIT1. J Hum Genet 2007;52:926-933.

24 Matsuoka S, Thompson JS, Edwards MC: Imprinting of the gene encoding a human cyclin-dependent kinase inhibitor, p57KIP2, on chromosome 11p15. Proc Natl Acad Sci USA 1996;93:3026-3030.

25 Li WC, Kuszak JR, Dunn K, Wang RR, Ma W, Wang GM: Lens epithelial cell apoptosis appears to be a common cellular basis for non-congenital cataract development in humans and animals. J Cell Biol 1995;130:169-181. 


\section{Cellular Physiology Cell Physiol Biochem 2017;42:295-305 \begin{tabular}{l|l} 
DOI: 10.1159/000477330 & O 2017 The Author(s). Published by S. Karger AG, Basel \\
www.karger.com/cpb
\end{tabular} \\ Jin et al.: Long Non-Coding RNA KCNQ1OT1 Promotes Cataractogenesis}

26 Ji Y, Rong X, Li D, Cai L, Rao J, Lu Y: Inhibition of Cartilage Acidic Protein 1 Reduces Ultraviolet B Irradiation Induced-Apoptosis through P38 Mitogen-Activated Protein Kinase and Jun Amino-Terminal Kinase Pathways. Cell Physiol Biochem 2016;39:2275-2286.

27 Fan S-h, Wang Y-y, Lu J, Zheng Y-l, Wu D-m: Luteoloside Suppresses Proliferation and Metastasis of Hepatocellular Carcinoma Cells by Inhibition of NLRP3 Inflammasome. PLoS ONE 2014;9:e89961.

28 Li YJ, Jiang Q, Cao GF, Yao J, Yan B: Repertoires of autophagy in the pathogenesis of ocular diseases. Cell Physiol Biochem 2015;35:1663-1676.

29 Penna E, Orso F, Cimino D, Tenaglia E, Lembo A, Quaglino E: microRNA- 214 contributes to melanoma tumour progression through suppression of TFAP2C. EMBO J 2011;30:1990-2007.

30 Worley LA, Long MD, Onken MD, Harbour JW: Micro-RNAs associated with metastasis in uveal melanoma identified by multiplexed microarray profiling. Melanoma Res 2008;18:184-190.

-31 Yang L, Ge D, Cao X, Ge Y, Chen H, Wang W, Zhang H: MiR-214 Attenuates Osteogenic Differentiation of Mesenchymal Stem Cells via Targeting FGFR1. Cell Physiol Biochem 2016;38:809-820.

32 Chen Q, Qin R, Fang Y, Li H, Liu Y: A Functional Variant at the miR-214 Binding Site in the Methylenetetrahydrofolatereductase Gene Alters Susceptibility to Gastric Cancer in a Chinese Han Population. Cell Physiol Biochem 2015;36:622-630.

-33 Sahoo S, Meijles DN, Al Ghouleh I: MEF2C-MYOCD and Leiomodin1 Suppression by miRNA-214 Promotes Smooth Muscle Cell Phenotype Switching in Pulmonary Arterial Hypertension. PLoS One 2016;11:e0153780.

34 Chen H, Lu Y, Cao Z: Cadmium induces NLRP3 inflammasome-dependent pyroptosis in vascular endothelial cells. Toxicol Lett 2016;246:7-16.

-35 Tuo YL, Li XM:Long noncoding RNA UCA1 modulates breast cancer cell growth and apoptosis through decreasing tumor suppressive miR-143. Eur Rev Med Pharmacol Sci 2015;19:3403-3411.

-36 Xue M,Pang H, Li X: Long non-coding RNA urothelial cancer-associated 1 promotes bladder cancer cell migration and invasion by way of the hsa-miR-145-ZEB1/2-FSCN1 pathway. Cancer Sci 2016;107:18-27. 